\title{
Fibromyalgia: evolving concepts over the past 2 decades
}

\author{
Mary-Ann Fitzcharles MB ChB, Peter A. Ste-Marie BA, John X. Pereira MD; for the Canadian Fibromyalgia \\ Guidelines Committee
}

$\mathrm{F}$ rom being a condition of suspect validity 2 decades ago, fibromyalgia is now much more widely accepted as a true syndrome, with pathogenesis centred in the nervous system and abnormalities shown in pain-regulating mechanisms at various levels of the central and peripheral nervous systems. ${ }^{1-3}$ The cause of fibromyalgia is unknown, but there is some evidence for a genetic predisposition, abnormalities in the stress response system or hypothalamic-pituitary axis, and possible triggering events. ${ }^{4}$ In the absence of physical findings or abnormal results from laboratory tests, clinicians must rely on the time-honoured art of medicine to diagnose fibromyalgia.

Fibromyalgia was recognized with the publication of the American College of Rheumatology classification criteria in 1990 , which were remodelled in 2010. ${ }^{5,6}$ Patients experience chronic widespread pain as the main symptom and often have associated symptoms of fatigue, nonrestorative sleep, cognitive dysfunction, mood disorder and other somatic symptoms, which are present to variable degrees in individual patients. ${ }^{7}$ Canadian prevalence rates are reported to be about $1 \%-5 \%$; the prevalence increases with age, and female patients are affected at least 6 times more often than male patients. ${ }^{8}$ Although seen most often in middleaged women, fibromyalgia has been described in men, children, teenagers and older people.

Fibromyalgia is a costly condition accentuated by delay in diagnosis. Health care costs in the province of Quebec were found to be more than $\$ 4000$ per year for patients with fibromyalgia, an amount $47 \%$ higher than costs for patients without the condition. ${ }^{9}$ The diagnosis does not require specialist confirmation, and ideal care for most patients will be in the primary care setting. Treatment must move away from the simple prescription of a drug, with incorporation of nonpharmacologic strategies in a multimodal approach. Because fibromyalgia persists over years, with symptoms fluctuating but seldom disappearing completely, patients should be encouraged to identify strategies that can modulate symptoms during a flare-up..$^{10}$ Finally, patient reports of functional impairment, often leading to work disability, require critical assessment with the concern that this diagnosis may be given too freely, resulting in negative health and socioeconomic effects.

This review is based primarily on the 2012 Canadian Guidelines for the Diagnosis and Management of Fibromyalgia Syndrome ${ }^{11}$ (Box $1^{11,12}$ ) and provides an overview of the changes in our understanding and management of fibromyalgia that have occurred over the past 2 decades.

\section{How has the diagnosis changed?}

In contrast to the original concept of fibromyalgia, which was entirely focused on the symptom of pain and the presence of tender points, the Fibromyalgia Syndrome Module of the 9th International Consensus on Outcome Measures in Rheumatology has defined a broader core set of domains that include fatigue, sleep disturbance, cognitive changes and mood disturbance, with the use of a Delphi exercise. ${ }^{7}$ This change in concept is reflected in the 2010 criteria of the American College of Rheumatology, which were specifically developed to identify study participants in the research setting and not for diagnosis of the condition in an individual patient in the clinic. ${ }^{6}$ Because a patient's symptoms will vary in severity over time, strict cut-offs to fulfill criteria should not be applied at a single point. Therefore, when making a diagnosis of fibromyalgia, the physician should perform a comprehensive clinical evaluation, with attention to all core symptom components, comorbidities and current use of medications (Box 23,4,13,14).

Chronic widespread pain remains the core
Competing interests: Mary-Ann Fitzcharles has received consulting fees, speaker fees and honoraria from Janssen, Eli Lilly, Pfizer, Purdue Pharma and Valeant Pharmaceuticals International. She has provided expert testimony for both plaintiffs and the defence in medicolegal adjudications, including insurance and workers' compensation cases concerning injuries that lead to various pain syndromes. Peter Ste-Marie is supported by a grant from the Louise and Alan Edwards

Foundation. John Pereira has received independently awarded research support from Pfizer Canada.

This article has been peer reviewed.

Correspondence to: Mary-Ann Fitzcharles, mary-ann.fitzcharles@muhc .mcgill.ca

CMAJ 2013. DOI:10.1503 /cmaj.121414

\section{- Ker points}

- Fibromyalgia is a clinical construct without any confirmatory clinical or laboratory test.

- Pain is the chief symptom, associated with sleep disturbance, fatigue, cognitive changes and mood disorder of variable severity.

- Primary care physicians can confidently diagnose fibromyalgia without the need for specialist confirmation, and the current practice of extensive investigations should be curtailed.

- Ideal management is a multimodal approach combining nonpharmacologic and pharmacologic treatments, with the understanding that fibromyalgia symptoms fluctuate over time and seldom completely disappear. 
symptom of fibromyalgia, reported as diffuse, fluctuating and with neuropathic features among some patients. Fatigue, which is tightly connected with sleep disorder, is present in more than $90 \%$ of patients with fibromyalgia, whereas abnormal sleep with prolonged sleep latency, sleep disturbance and fragmented sleep occurs in up to $75 \%$ of patients. ${ }^{7,15}$ Cognitive dysfunction is associated with pain and includes poor working memory, free recall and verbal fluency, and spatial memory alterations. ${ }^{16}$ Mood disorder, including depression, anxiety or both, is present in up to $75 \%$ of patients with fibromyalgia. ${ }^{17}$ Fibromyalgia is now recognized as a polysymptomatic distress syndrome. Other conditions causing pain can occur concomitantly, including irritable bowel syndrome, migraine and dysmenorrhea. ${ }^{18}$ Patients may also experience lower urinary tract symptoms, myofascial pain involving the face and temporomandibular pain. ${ }^{18}$

In the past, tender points were synonymous with fibromyalgia, with the tender point count embedded in the diagnosis. The value of this subjective physical finding has been debated because of variable reliability, poor association with symptom severity and the ability to be faked for dishonest reasons. ${ }^{19,20}$ The weight attributed to the tender point count, a finding previously used erroneously to confirm or reject a diagnosis, has detracted from the clinical concept of fibromyalgia and was thus revisited in the 2010 American College of Rheumatology criteria and the 2012 Canadian Fibromyalgia Guidelines. ${ }^{6,11}$

How then should physicians diagnose fibro-

\section{Box 1: Evidence used in this review ${ }^{11,12}$}

This review is based on a literature search used to develop the 2012 Canadian Guidelines for the Diagnosis and Management of Fibromyalgia Syndrome, endorsed by the Canadian Pain Society and the Canadian Rheumatology Association." After a needs assessment with input from 139 Canadian health care professionals representing various disciplines, 18 key questions drove a comprehensive literature search, which was conducted at the McGill University Health Sciences Library. Embase, MEDLINE, PsycINFO and the Cochrane Library were searched for literature published from Jan. 1, 1990, to July 31, 2010. After exclusion and merger of the original 4735 articles, 363 articles were further screened by their abstracts. Of these, 150 were suitable for formal attribution of a level of evidence, and full texts were accessed. Grading of the evidence was based on the grading system of the Oxford Centre for Evidence Based Medicine. ${ }^{12}$ Recommendations were drafted, assigned a level of evidence, graded by the executive committee and submitted to a panel of 35 health care professionals, who voted on acceptance and provided input regarding modification. Of the original 60 recommendations, 49 achieved $80 \%$ approval after a first pass. The 11 remaining recommendations were modified according to suggestions and submitted to a second vote, and all achieved approval at the second vote. The entire document was reviewed by an international expert, and further reviewed by the Canadian Pain Society using the AGREE II Score Sheet guideline appraisal tool (Appraisal of Guidelines for Research and Evaluation, agreetrust.org), with resulting shortening of the guideline document and combination, but not elimination, of recommendations to reduce the total number from 60 to 46 . myalgia (Box 23,4,73,14)? Fibromyalgia can be positively diagnosed in the primary care setting as a polysymptomatic distress syndrome with a composite of widespread pain and associated symptoms. There is no need for extensive laboratory and radiographic investigation, and it is not a diagnosis of exclusion. In the absence of a confirmatory test, expert opinion suggests that investigations should be limited to simple blood tests, including a complete blood count, erythrocyte sedimentation rate, C-reactive protein level, thyroid function and creatine kinase level, when an alternative diagnosis is considered. ${ }^{14}$ Any additional testing should be driven by the clinical presentation, and excessive testing should be limited. The tender point count should not be used to confirm a diagnosis of fibromyalgia.

Although rheumatologists previously had ownership of this condition, the considerable number of people affected makes routine confirmation by specialists untenable. ${ }^{21}$ Only patients with a particular challenge in management or in whom symptoms may suggest an alternative diagnosis may require evaluation by a specialist. Excessive and repeated investigation may cause patient uncertainty and foster poor healthrelated behaviours such as passivity and dependence on health professionals.

\section{How does new neurophysiologic evidence translate into rational management?}

Neurophysiologic studies have identified abnormalities of pain processing at various levels in the nervous system in patients with fibromyalgia, although there is to date no single change unique to fibromyalgia. ${ }^{3}$ Changes include peripheral sensitization at the primary somatosensory neuron,

Box 2: How to diagnose fibromyalgia ${ }^{3,4,7,13,14}$

- The diagnosis is made on the basis of a composite of symptoms, centred on chronic widespread pain and absence of physical findings that would indicate another condition.

- Clinical evaluation should include a history of physical and mental health, with attention to the patient's psychosocial context, because these factors may influence the expression of fibromyalgia.

- A physical examination is required for all patients, and findings may be completely within normal limits.

- Examination for tender points is not required to confirm the diagnosis.

- Only limited laboratory testing should be conducted for most patients. 
central sensitization at the level of the dorsal horn, changes at the level of the thalamus and brain gray matter, and impairment of the natural pain inhibition mediated via descending pathways from the brainstem to the dorsal horn of the spinal cord, termed diffuse noxious inhibitory control. ${ }^{1,4}$ In the past, treatments for fibromyalgia were guided by the notion that improved sleep by use of tricyclic antidepressants would improve symptoms, whereas current understanding points to changes in nerve transmission as the mechanism of action for most agents. ${ }^{4}$ In this context, treatments focused on neuropathy that also include nonpharmacologic strategies harnessing endogenous pain modulatory systems are rational. Therefore, the shift from considering fibromyalgia as a soft-tissue disorder with pain driven by traditional inflammatory molecules to seeing it as a neuropathic process opens the door for treatment strategies that may have more directed impact on neuropathic pain.

Pain-relieving treatments may be simplistically categorized as those that dampen incoming ascending pain signals and those that augment descending pain inhibitory mechanisms..$^{22}$ Reduction of incoming pain signals at the peripheral nerve fibre may be achieved with use of antiinflammatory agents and opioids, as well as physical interventions such as massage and application of heat or cold. Strategies with impact on dorsal horn mechanisms that mediate sensitization of pain signals include use of anticonvulsants, opioids and cannabinoids, as well as physical interventions such as massage, transcutaneous electrical nerve stimulation and acupuncture.

Nonpharmacologic interventions such as relaxation, distraction, exercise and cognitive behavioural training play an important role in modulating descending pain mechanisms with signals originating at the level of the cerebral cortex. Finally, the descending pathways may be affected by antidepressant drugs that increase the level of the transmitters serotonin and norepinephrine at the synapse, thereby affecting voltage-gated calcium channels. Agents in the opioid and cannabinoid classes act at many levels in the nervous system, affecting both the sensory and emotional components of pain.

Therefore, treatments focused on the symptoms of fibromyalgia have different specific mechanisms of action but mostly interfere with neurotransmitter release and uptake. ${ }^{4}$ Given the complexity of the pain experience in fibromyalgia, a multimodal strategy that is tailored to the patient is most effective. Treatments should therefore incorporate nonpharmacologic strategies with selected drug therapy, but without reliance on a single "ideal" drug.

\section{What is the ideal treatment strategy?}

Because there is no cure for fibromyalgia, the goal of treatment is to improve symptoms and optimize function (Box $3^{47,8,23-30}$ ). Symptom-based management will take into account the heterogeneous nature of fibromyalgia with attention to all components of suffering. ${ }^{25}$ The ideal strategy will combine nonpharmacologic and pharmacologic treatments in a multimodal approach tailored to the individual patient. ${ }^{31}$ Physicians are therefore

\footnotetext{
Box 3: Treatment of fibromyalgia ${ }^{4,7,8,23-30}$

General concepts

- No single treatment outperforms others, so a multimodal approach is best.

- The outcome goal is symptom control and improved function.

- Patient engagement improves adherence, and treatment failure is associated with passivity, uncontrolled mood disorder, catastrophizing and secondary gain.

\section{Nonpharmacologic treatment}

- It is essential that nonpharmacologic therapies be incorporated in a treatment plan.

- Coping skills, self-efficacy and patient education form the foundation of self-management.

- Regular exercise should be encouraged for all patients, even though the evidence is currently limited.

- There is insufficient evidence for recommendation of complementary and alternative medicine treatments.

Pharmacologic treatment

- Symptom-based management should address individual symptoms of pain, sleep, mood and fatigue, but effects may be modest.

- A "start low, go slow" approach is recommended when starting pharmacologic therapy.

- Drugs with different mechanisms of action may be combined to improve efficacy.

- Throughout treatment, the efficacy and adverse-effect profile should be monitored.
} 
encouraged to identify the most troublesome symptom(s) and direct management accordingly. With the knowledge that symptoms persist over time, most patients will identify strategies that can modulate exacerbations. ${ }^{10}$ Patients must be encouraged to be active participants in their health care management, because self-efficacy (the belief in one's capabilities to achieve a goal or an outcome) and adherence to treatment predict improved outcome, whereas a less favourable outcome is associated with external locus of control (the belief that only external factors can influence one's health, as opposed to an internal locus of control, which is the belief that one has control over one's own health) and personality traits such as neuroticism and catastrophizing. ${ }^{26,27}$

Current evidence indicates that there is no single ideal treatment, with responses modest at best. ${ }^{25}$ Interpretation of treatment effects in clinical trials may be confounded in the absence of a universally accepted outcome measure, with studies variably reporting effects on pain, quality of life or other factors affected by fibromyalgia.

In a network meta-analysis that examined the comparative efficacy of pharmacologic and nonpharmacologic treatments, the effects of most treatments were calculated to be small to moderate, with reduced effect size for most treatments as sample sizes increased. ${ }^{28}$ When studies with more than 50 participants per group were examined, the standardized mean differences (SMDs) with $95 \%$ credibility intervals (CrIs), interpreted similarly to confidence intervals (CIs), ranged from -0.19 (95\% CrI -0.60 to 0.23$)$ for treatment with selective serotonin reuptake inhibitors, indicating no significant effect, to $-0.47(95 \%$ CrI -0.69 to -0.24 ) for treatments of multicomponent therapy. An SMD of -0.20 indicates small differences between groups, whereas an SMD of -0.80 indicates a large effect of a treatment. There is therefore a need for larger, wellcontrolled trials to help direct treatments.

\section{Nonpharmacologic treatments}

Nonpharmacologic strategies form an integral part of multicomponent therapy, which currently has the best evidence for effect in treatment of fibromyalgia. These interventions can include education to improve a patient's self-efficacy and coping mechanisms, as well as exercise, psychological interventions such as cognitive behavioural therapy, and multidisciplinary therapy. Nonpharmacologic interventions should be incorporated into every patient's treatment plan (Box 3). 7,8,28,29 Taking into consideration the global health benefits associated with a regular exercise program, it is intuitive that exercise should be a normal routine for all patients with fibromyalgia. The evidence for exercise effects on specific symptoms of fibromyalgia is not convincing because of heterogeneous study design, variable outcome measurements and different modalities of exercise examined. In a metaanalysis of 45 studies, 10 of which were eligible for inclusion, short-term improvements in pain were seen with exercise, which included aerobic, strength-training, pool-based and multicomponent exercise. ${ }^{32}$ However, the need for long-term studies was identified. ${ }^{32}$ When at least 50 participants were included per group and quality of life was the outcome measure, the SMD for aerobic exercise was -0.28 (95\% CrI -0.54 to 0.01 ), indicating no significant effect. ${ }^{28}$

Cognitive behavioural therapy was found to help patients cope with pain by improving painrelated behaviours, self-efficacy and overall physical functioning, but effects on symptoms were not significant. ${ }^{33}$ The SMD for the effect of cognitive behavioural therapy on pain was calculated to be -0.43 (95\% $\mathrm{CrI}-0.74$ to -0.12 ) and -0.55 for quality of life $(95 \% \mathrm{CrI}-0.96$ to $-0.15)$, indicating a moderate effect. ${ }^{28}$

Multicomponent treatment that includes either educational or psychological therapy combined with at least 1 exercise program was found to be effective in the short term for reduction of pain (SMD $-0.37,95 \% \mathrm{CI}-0.62$ to -0.13 ), fatigue (weighted mean difference $-0.85,95 \%$ CI -1.50 to -0.20 ), symptoms of depression (SMD $-0.67,95 \%$ CI -1.08 to -0.26 ) and limitations to health-related quality of life (SMD $-0.59,95 \%$ CI -0.90 to -0.27 ), while improving physical fitness (SMD 0.30, 95\% CI 0.02 to 0.57). However, only the positive effects related to physical fitness were maintained in the long term (SMD 0.30, 95\% CI 0.09 to 0.51$){ }^{24}$ Although patients with fibromyalgia commonly use complementary medicines, including ingested and practitioner-administered medicines, there is currently insufficient evidence to recommend these therapies. ${ }^{29}$ Taking cost containment into account, access to a nurse for health-related advice and support, education sessions and participation in a group exercise program are modalities accessible to most patients.

\section{Pharmacologic treatments}

Most patients with fibromyalgia use at least 2 medications for management of symptoms, in doses lower than those used in clinical trials (Box 3). 4,25,28,30 Because patients frequently report considerable sensitivity to pharmacologic treatment, expert consensus recommends that medications be started in low doses with gradual upward titration, and with consideration of combination of drugs with different mechanisms of action. ${ }^{30}$ 
Traditional pain-relieving drugs such as simple analgesics, nonsteroidal anti-inflammatory drugs (NSAIDs) and opioids other than tramadol have had limited study in the treatment of fibromyalgia. In view of the toxicity of NSAIDs, these drugs should be used in low doses and for short periods. Up to $30 \%$ of patients with fibromyalgia in North America use opioids, ${ }^{34}$ and patients believe that these agents give the best effect. ${ }^{35}$ However, in the absence of evidence for use in the treatment of fibromyalgia and with concerns regarding safety, NSAIDs and particularly opioids should be used with extreme caution.

Two drug classes that affect neuropathic pain are the gabapentinoids with effect on sensitization, and antidepressants that modulate serotonin and norepinephrine, molecules important for diffuse noxious inhibitory control. Pregabalin and duloxetine, respectively belonging to the aforementioned classes, have received Health Canada approval for treatment of fibromyalgia pain.

Because of the adverse-effect profile of tricyclic antidepressants, evaluation of other antidepressants has been prompted. In a systematic review of 26 studies evaluating the use of antidepressants in patients with fibromyalgia (13 studies evaluated amitriptyline, 12 evaluated selective serotonin reuptake inhibitors [5 paroxetine, 4 fluoxetine, 2 citalopram, 1 sertraline] and 3 evaluated serotonin norepinephrine reuptake inhibitors [2 duloxetine, 1 milnacipran]), all agents with the exception of citalopram showed a positive effect on pain and on other features of fibromyalgia, including fatigue, depression, abnormal sleep and poor quality of life. ${ }^{36}$ In a subsequent meta-analysis by the same group examining 18 randomized controlled trials with a median duration of 8 weeks, the effect size for pain reduction was largest for tricyclic antidepressants, with selective serotonin reuptake inhibitors and serotonin norepinephrine reuptake inhibitors showing smaller effects. ${ }^{37}$ Only the serotonin norepinephrine reuptake inhibitors have been examined in studies with at least 100 participants per group, with the SMD for pain and quality of life reported as $-0.26(95 \%$ $\mathrm{CrI}-0.35$ to -0.19$)$ and $-0.21(95 \% \mathrm{CrI}$ -0.29 to -0.14$)$, respectively, indicating a modest effect. $^{28}$

Gabapentinoids, classified as second-generation anticonvulsants, have shown clinical efficacy in the treatment of fibromyalgia, although the true effect may be small, with only a minority of patients having substantial benefit. ${ }^{38-40}$ In an analysis of 127 randomized trials of gabapentin and pregabalin, with 5 studies included for metaanalysis, there was evidence for reduction of pain, improved sleep and quality of life, but effect sizes for active treatment were in the low range. The following values were reported: reduced pain $(\mathrm{SMD}-0.28,95 \% \mathrm{CI}-0.36$ to -0.20 ), improved sleep (SMD -0.39, 95\% CI -0.48 to -0.39$)$ and improved quality of life (SMD $-0.30,95 \% \mathrm{CI}-0.46$ to -0.15$)$. $^{38}$

As symptoms of fibromyalgia wax and wane, drug treatments may be adjusted accordingly, with less use during periods of quiescence, with the exception of antidepressants, which mostly require a more gradual reduction. Care is required to monitor continued efficacy of any pharmacologic treatment, with attention to adverse effects that may mimic fibromyalgia.

\section{What outcome measures can be applied in clinical practice?}

In view of the diversity of subjective symptoms among patients with fibromyalgia, the most meaningful measure of clinical outcome has not been clearly defined. Study authors may confound the clinician by choosing individual measures, such as pain reduction, improved sleep, fatigue or quality of life, to advocate for a specific treatment. Although questionnaires such as the Fibromyalgia Impact Questionnaire, the Revised Fibromyalgia Impact Questionnaire, the Stanford Health Assessment Questionnaire and others measuring pain, sleep, fatigue and depression have been applied in the research setting, they are generally cumbersome, with no single questionnaire showing accuracy or validity to reflect change over time. ${ }^{41-43}$ The tender point count is not a clinically relevant or reliable outcome measurement and should not be used.

The clinician needs a simple and easily applicable measurement tool for use in daily practice. A change in clinical status measured by a 7 -point Likert scale $(1=$ much worse, 7 = much better) has shown validity equivalent to more extensive questionnaires and can be recommended for clinical use. ${ }^{44}$ Documenting patient goals and levels of achievement is a practical strategy, with concrete meaning. Taking into account fluctuation of symptoms that tend to persist over time, outcome at any one point should not be expected to be finite.

\section{What are the challenges regarding work status and disability insurance?}

Patients with fibromyalgia often report considerable functional impairment, which extends to the 
contentious issue of work disability. Impairment in fibromyalgia, which may be greater than impairment in rheumatoid arthritis, ${ }^{45}$ is difficult to reconcile with a mostly healthy-looking individual. The report that up to one-third of people with fibromyalgia are receiving disability compensation is worrisome in the context of a condition that presents with subjective symptoms that cannot be verified. ${ }^{46}$ There is concern that, for some, the diagnostic label may infer disability entitlement.

In that symptoms of fibromyalgia are entirely subjective, they can be faked for dishonest reasons. When results of testing for effort in cognitive function were examined, one-third of patients with fibromyalgia receiving or seeking disability benefits failed an effort test, designed to identify exaggeration of cognitive impairment, compared with only $4 \%$ of patients with fibromyalgia who were not in a secondary-gain setting. ${ }^{47}$ Therefore, physicians, particularly those adjudicating disability issues, must remain empathetic, but alert and cautious. In addition, although the cause of fibromyalgia remains uncertain, the legal system has allowed for triggering events to be determined as having caused fibromyalgia. ${ }^{48}$ In this context, physicians should be cautious when attributing the cause of fibromyalgia to an injury, particularly in the workplace, because this determination may have socioeconomic consequences. Physicians should evaluate the global presentation of the patient, with particular attention to the physical and psychosocial status before the injury.

Maintaining a life pattern as close to normal as possible is intuitively desirable. Patients with fibromyalgia who are in the workforce have generally less severe symptoms and better quality of life than patients who are unemployed, and they maintain this better health status over time. ${ }^{49} \mathrm{In}$ an Internet survey of 2580 patients with fibromyalgia, higher physical function was associated with younger age, higher education level, less fatigue, less medication use and more exercise..$^{50}$ Treatment strategies addressing the variables that can be changed may improve function.

Patients with fibromyalgia who had a negative perception about pain and had greater perceived functional disability were less likely to return to work after a multidisciplinary rehabilitation program. ${ }^{51}$ Similarly, patients' perceived physical limitations predicted employment status better than affective symptoms or pain. ${ }^{52}$ Therefore, strategies aimed at improving coping skills and implementing an internal locus of control will likely facilitate return to work.

Pacing, whereby patients are encouraged to modulate activity to avoid bouts of overexertion that can result in exhaustion, can improve function. When applied to the workforce, this may facilitate retention in employment. ${ }^{53}$ Specific reasonable adjustments within the working environment may be helpful. Although the physical and psychological requirements of a job influence employment, the total life situation and ability to influence work parameters contribute to continued employment. ${ }^{54}$

\section{Gaps in knowledge}

Until the pathogenesis of fibromyalgia has been more clearly established, skepticism about the condition will remain. Even within the limitations of current knowledge, physicians must care for these patients according to the best available evidence and using sound clinical judgment. Although the diagnosis of fibromyalgia is currently based entirely on a clinical evaluation, physicians can feel confident in making a diagnosis without the need to resort to extensive investigations and specialist referral for most patients. Improved understanding of fibromyalgia has already opened the door to more diverse treatment options. However, because treatments offer only a modest effect at best, patients and physicians may experience frustration. Because there is no cure for fibromyalgia, research on more effective treatment modalities is needed.

An important research question germane to both fibromyalgia and chronic pain is whether early diagnosis and intervention may reduce the severity and duration of symptoms. The true rehabilitation potential for patients with prolonged disability from fibromyalgia is also unknown and worthy of study. Finally, long-term cohort studies may improve the understanding of the natural history of this condition.

More details on the diagnosis and management of fibromyalgia can be found in the 2012 Canadian Guidelines for the Diagnosis and Management of Fibromyalgia Syndrome. ${ }^{11}$ The full guidelines, endorsed by the Canadian Pain Society and Canadian Rheumatology Association, are available at www.fmguidelines.ca.

\section{References}

1. Price DD, Staud R. Neurobiology of fibromyalgia syndrome. J Rheumatol Suppl 2005;75:22-8.

2. Sommer C, Häuser W, Burgmer M, et al. Etiology and pathophysiology of fibromyalgia syndrome [article in German]. Schmerz 2012;26:259-67.

3. Ceko M, Bushnell MC, Gracely RH. Neurobiology underlying fibromyalgia symptoms. Pain Res Treat 2011; Oct. 27 [Epub ahead of print].

4. Perrot S, Dickenson AH, Bennett RM. Fibromyalgia: harmonizing science with clinical practice considerations. Pain Pract 2008;8:177-89.

5. Wolfe F, Smythe HA, Yunus MB, et al. The American College of Rheumatology 1990 Criteria for the Classification of Fibromyalgia. Report of the Multicenter Criteria Committee. Arthritis 
Rheum 1990;33:160-72.

6. Wolfe F, Clauw DJ, Fitzcharles M-A, et al. The American College of Rheumatology Preliminary Diagnostic Criteria for Fibromyalgia and Measurement of Symptom Severity. Arthritis Care Res (Hoboken) 2010;62:600-10.

7. Mease P, Arnold LM, Choy EH, et al. Fibromyalgia syndrome module at OMERACT 9: domain construct. J Rheumatol 2009; 36:2318-29.

8. McNally JD, Matheson DA, Bakowsky VS. The epidemiology of self-reported fibromyalgia in Canada. Chronic Dis Can 2006; 27:9-16.

9. Lachaine J, Beauchemin C, Landry P-A. Clinical and economic characteristics of patients with fibromyalgia syndrome. Clin J Pain 2010;26:284-90.

10. Walitt B, Fitzcharles MA, Hassett AL, et al. The longitudinal outcome of fibromyalgia: a study of 1555 patients. J Rheumatol 2011;38:2238-46.

11. Fitzcharles M-A, Ste-Marie PA, Goldenberg DL, et al. Canadian Guidelines for the Diagnosis and Management of Fibromyalgia Syndrome: executive summary. Pain Res Manag. In press.

12. Oxford Centre for Evidence-Based Medicine Levels of Evidence Working Group. The Oxford 2011 Levels of Evidence. Oxford (UK): Oxford Centre for Evidence-Based Medicine. Available: www.cebm.net/index.aspx?o=5653 (accessed 2013 Jan. 20).

13. Wolfe F, Hauser W. Fibromyalgia diagnosis and diagnostic criteria. Ann Med 2011;43:495-502.

14. Goldenberg DL. Diagnosis and differential diagnosis of fibromyalgia. Am J Med 2009;122(Suppl 12):S14-21.

15. Moldofsky $\mathrm{H}$. The significance of the sleeping-waking brain for the understanding of widespread musculoskeletal pain and fatigue in fibromyalgia syndrome and allied syndromes. Joint Bone Spine 2008;75:397-402.

16. Park DC, Glass JM, Minear M, et al. Cognitive function in fibromyalgia patients. Arthritis Rheum 2001;44:2125-33.

17. Epstein SA, Kay G, Clauw D, et al. Psychiatric disorders in patients with fibromyalgia. A multicenter investigation. Psychosomatics 1999; 40:57-63.

18. Pöyhiä R, Da Costa D, Fitzcharles MA. Previous pain experience in women with fibromyalgia and inflammatory arthritis and nonpainful controls. J Rheumatol 2001;28:1888-91.

19. Harth M, Nielson WR. The fibromyalgia tender points: Use them or lose them? A brief review of the controversy. J Rheumatol 2007;34:914-22.

20. Khostanteen I, Tunks ER, Goldsmith $\mathrm{CH}$, et al. Fibromyalgia: Can one distinguish it from simulation? An observer-blind controlled study. J Rheumatol 2000;27:2671-6.

21. Shir Y, Fitzcharles MA. Should rheumatologists retain ownership of fibromyalgia? J Rheumatol 2009;36:667-70.

22. Dickenson A. Pain and its control. Curr Med Res Opin 2011;27: 2075-8.

23. Goldenberg DL, Burckhardt C, Crofford L. Management of fibromyalgia syndrome. JAMA 2004;292:2388-95.

24. Häuser W, Bernardy K, Arnold B, et al. Efficacy of multicomponent treatment in fibromyalgia syndrome: a meta-analysis of randomized controlled clinical trials. Arthritis Rheum 2009;61: 216-24.

25. Boomershine CS, Crofford LJ. A symptom-based approach to pharmacologic management of fibromyalgia. Nat Rev Rheumatol 2009;5:191-9.

26. Dobkin PL, Liu A, Abrahamowicz M, et al. Predictors of disability and pain six months after the end of treatment for fibromyalgia. Clin J Pain 2010;26:23-9.

27. Martínez MP, Sanchez AI, Miro E, et al. The relationship between the fear-avoidance model of pain and personality traits in fibromyalgia patients. J Clin Psychol Med Settings 2011;18: 380-91.

28. Nüesch E, Hauser W, Bernardy K, et al. Comparative efficacy of pharmacological and non-pharmacological interventions in fibromyalgia syndrome: network meta-analysis. Ann Rheum Dis 2013:72;955-62.

29. De Silva V, El-Metwally A, Ernst E, et al. Evidence for the efficacy of complementary and alternative medicines in the management of fibromyalgia: a systematic review. Rheumatology (Oxford) 2010;49:1063-8.

30. Goldenberg DL, Clauw DJ, Fitzcharles MA. New concepts in pain research and pain management of the rheumatic diseases. Semin Arthritis Rheum 2011;41:319-34.

31. Rossy LA, Buckelew SP, Dorr N, et al. A meta-analysis of fibromyalgia treatment interventions. Ann Behav Med 1999;21: 180-91.

32. Ramel J, Bannuru R, Griffith M, et al. Exercise for fibromyalgia pain: a meta-analysis of randomized controlled trials. Curr Rheumatol Rep 2009;5:188-93.

33. Bernardy K, Fuber N, Kollner V, et al. Efficacy of cognitive- behavioral therapies in fibromyalgia syndrome - a systematic review and metaanalysis of randomized controlled trials. J Rheumatol 2010;37:1991-2005.

34. Fitzcharles MA, Ste-Marie PA, Gamsa A, et al. Opioid use, misuse, and abuse in patients labeled as fibromyalgia. Am J Med 2011;124:955-60.

35. Bennett RM, Jones J, Turk DC, et al. An internet survey of 2,596 people with fibromyalgia. BMC Musculoskelet Disord 2007; 8:27.

36. Uçeyler N, Häuser W, Sommer C. A systematic review on the effectiveness of treatment with antidepressants in fibromyalgia syndrome. Arthritis Rheum 2008;59:1279-98

37. Häuser W, Bernardy K, Uceyler N, et al. Treatment of fibromyalgia syndrome with antidepressants: a meta-analysis. JAMA 2009;301:198-209.

38. Häuser W, Bernardy K, Uceyler N, et al. Treatment of fibromyalgia syndrome with gabapentin and pregabalin - a metaanalysis of randomized controlled trials. Pain 2009;145:69-81.

39. Siler AC, Gardner H, Yanit K, et al. Systematic review of the comparative effectiveness of antiepileptic drugs for fibromyalgia. J Pain 2011;12:407-15

40. Moore RA, Straube S, Wiffen PJ, et al. Pregabalin for acute and chronic pain in adults. Cochrane Database Syst Rev 2009;(3): CD007076.

41. Bennett RM, Friend R, Jones KD, et al. The Revised Fibromyalgia Impact Questionnaire (FIQR): validation and psychometric properties. Arthritis Res Ther 2009;11:R120.

42. Burckhardt CS, Clark SR, Bennett RM. The Fibromyalgia Impact Questionnaire: development and validation. J Rheumatol 1991;18:728-33.

43. Bruce B, Fries JF. The Stanford Health Assessment Questionnaire: dimensions and practical applications. Health Qual Life Outcomes 2003;1:20.

44. Geisser ME, Clauw DJ, Strand V, et al. Contributions of change in clinical status parameters to Patient Global Impression of Change (PGIC) scores among persons with fibromyalgia treated with milnacipran. Pain 2010;149:373-8.

45. Hidding A, van Santen M, De Klerk E, et al. Comparison between self-report measures and clinical observations of functional disability in ankylosing spondylitis, rheumatoid arthritis and fibromyalgia. J Rheumatol 1994;21:818-23.

46. Wolfe F, Anderson J, Harkness D, et al. Work and disability status of persons with fibromyalgia. J Rheumatol 1997;24:1171-8.

47. Gervais RO, Russell AS, Green P, et al. Effort testing in patients with fibromyalgia and disability incentives. J Rheumatol 2001; 28:1892-9.

48. Fitzcharles MA, Ste-Marie PA, Shir Y. A medicolegal analysis of worker appeals for fibromyalgia as a compensable condition following workplace soft-tissue injury. J Rheumatol 2013;40:323-

49. Reisine S, Fifield J, Walsh S, et al. Employment and health status changes among women with fibromyalgia: a five-year study. Arthritis Rheum 2008;59:1735-41.

50. Rutledge DN, Jones K, Jones CJ. Predicting high physical function in people with fibromyalgia. J Nurs Scholarsh 2007;39:31924.

51. Torres X, Collado A, Arias A, et al. Pain locus of control predicts return to work among Spanish fibromyalgia patients after completion of a multidisciplinary pain program. Gen Hosp Psychiatry 2009;31:137-45

52. Kurtze N, Gundersen KT, Svebak S. The impact of perceived physical dysfunction, health-related habits, and affective symptoms on employment status among fibromyalgia support group members. J Musculoskeletal Pain 2001;9:39-53.

53. Henriksson C, Burckhardt C. Impact of fibromyalgia on everyday life: a study of women in the USA and Sweden. Disabil Rehabil 1996;18:241-8.

54. Henriksson CM, Liedberg GM, Gerdle B. Women with fibromyalgia: work and rehabilitation. Disabil Rehabil 2005;27:68594.

Affiliations: Division of Rheumatology (Fitzcharles), McGill University; Alan Edwards Pain Management Unit (Fitzcharles, Ste-Marie), McGill University Health Centre; Faculty of Law (Ste-Marie), Université de Montréal, Montréal, Que.; and Faculty of Medicine (Pereira), University of Calgary, Calgary, Alta.

Contributors: All of the authors analyzed and interpreted the data, contributed to the writing and revising of the manuscript and gave final approval of the version submitted for publication. 